\title{
Review
}

\section{Epithelial cell polarity: a major gatekeeper against cancer?}

\author{
C Royer ${ }^{1}$ and $X L^{\star, 1}$
}

The correct establishment and maintenance of cell polarity are crucial for normal cell physiology and tissue homeostasis. Conversely, loss of cell polarity, tissue disorganisation and excessive cell growth are hallmarks of cancer. In this review, we focus on identifying the stages of tumoural development that are affected by the loss or deregulation of epithelial cell polarity. Asymmetric division has recently emerged as a major regulatory mechanism that controls stem cell numbers and differentiation. Links between cell polarity and asymmetric cell division in the context of cancer will be examined. Apical-basal polarity and cell-cell adhesion are tightly interconnected. Hence, how loss of cell polarity in epithelial cells may promote epithelial mesenchymal transition and metastasis will also be discussed. Altogether, we present the argument that loss of epithelial cell polarity may have an important role in both the initiation of tumourigenesis and in later stages of tumour development, favouring the progression of tumours from benign to malignancy.

Cell Death and Differentiation (2011) 18, 1470-1477; doi:10.1038/cdd.2011.60; published online 27 May 2011

Epithelial tissues are widely distributed, lining the external and internal surfaces of our bodies and playing a number of specialised roles. Each specialist function is achieved by the distinct structural organisation of epithelial cells within those tissues. Consequently, the integrity of their architecture is crucial. The majority of human cancers are derived from epithelial tissues, and display loss of cell polarity and often, as a consequence, tissue disorganisation.

Although the tumour suppressive function of polarity complexes is well established in Drosophila, it remains unclear whether a loss of cell polarity is a consequence or cause of human cancers. However, it is emerging that epithelial cell polarity may exert a tumour suppressive function in mammals through its participation in the establishment and maintenance of the three dimensional organisation of epithelial tissues as a whole. This theory is supported by the findings that polarity proteins are cellular targets of oncogenes, and an increasing list of tumour suppressors has been shown to regulate polarity pathways.

Functionally, apical-basal polarity has two fundamental roles in epithelial cells that are intimately linked to tumour suppression: (1) the regulation of asymmetric cell division and (2) the maintenance of the apical junctional complex (AJC). In epithelial stem cells, polarity proteins control asymmetric cell division by regulating the polarised localisation of cell fate determinants and the correct orientation of mitotic spindles. As a result, asymmetric cell division has a fundamental role in the control of progenitor or stem cell numbers and differentiation.
This is of particular interest in the context of the cancer stem cell theory, as a shift from asymmetric division of epithelial stem cells or cancer-initiating cells towards symmetric divisions would result in dedifferentiation on one hand and an increase in cancer-initiating cells on the other. Thus, a defect in asymmetric division could contribute to the emergence of tumours. As a result, over the last few years, there has been increasing interest in the identification of core cellpolarity mechanisms that govern the asymmetric division of epithelial stem cells, and understanding how their disruption may contribute to the development of cancer.

In addition to their role in the prevention of tumour initiation, core epithelial cell polarity mechanisms may also constitute a barrier to tumour metastasis and malignancy through their close connection to the AJC. The AJC encompasses tight and adherens junction complexes, and its structure is dependent on the integrity of the apical and basolateral polarity complexes. The loss of one of the key components of adherens junctions, E-cadherin, often occurs in later stages of tumourigenesis and is thought to contribute to epithelial mesenchymal transition (EMT), which represents a crucial step in metastasis. The importance of the AJC in suppressing cancer malignancy is supported by cancer genome sequencing data, which show that a large number of AJC components are frequently mutated in human cancers. In this review, we will therefore discuss whether a loss or deregulation of epithelial cell polarity favours tumour initiation, or is responsible for later stages of tumour development and malignancy.

\footnotetext{
${ }^{1}$ Ludwig Institute for Cancer Research, Old Road Campus Research Building, University of Oxford, Headington, Oxford OX3 7DQ, UK

${ }^{*}$ Corresponding author: X Lu, Ludwig Institute for Cancer Research, Old Road Campus Research Building, University of Oxford, Headington, Oxford OX3 7DQ, UK. Tel: + 44186561 7505; Fax: + 44186561 7504; E-mail: xin.lu@ludwig.ox.ac.uk

Keywords: cell polarity; cancer; asymmetric cell division; EMT; Par; scribble

Abbreviations: EMT, epithelial mesenchymal transition; AJC, apical junctional complex; aPKC, atypical protein kinase C; Lgl, lethal giant larvae; Dlg, Disc large; APC, adenomatous polyposis coli; ZO-1, zonula occludens-1 protein; PDZ, post synaptic density protein 95 (PSD95), Dlg and ZO-1

Received 17.2.11; revised 11.4.11; accepted 11.4.11; Edited by G Melino; published online 27.5.11
} 
The Main Players in the Establishment and Maintenance of Epithelial Cell Polarity

In order to understand how cells become disorganised in tumours it is vital to understand the key players and regulators that control and maintain cell polarity, which lead to epithelial tissue organisation. As expected, the molecular complexes involved in the establishment and maintenance of cell polarity are largely conserved throughout the metazoa, despite the wide range of epithelial tissue types and biological processes that require them. Three major complexes involved in the regulation of apical-basal cell polarity of epithelial cells have been described: The Crumbs-Pals1 (Stardust)-Patj-Lin-7 (Crumbs complex) and Par3 (Bazooka)-Par6-aPKC (Par complex) complexes, which are found apically, and the lethal giant larvae (Lgl)-Scribble (Scrib)-Disc large (Dlg) proteins (Scribble complex) that localise at the basolateral membrane. ${ }^{1-3}$ Both the Par and Crumbs complexes promote apical membrane identity, whereas the Scribble complex promotes basolateral membrane identity by antagonising the other two (Figure 1).

Among the three polarity complexes, the Par complex is the best studied in the context of apical-basal polarity in epithelial cells. Genetic screens in $C$. elegans have identified six Par genes (Par1-6). Classically, the proteins considered to be the core of the Par complex include the two Par PDZ domaincontaining proteins Par3 and Par6, with the addition of atypical protein kinase $\mathrm{C}(\mathrm{aPKC})$, and the Par complex has been shown to be required for the establishment and the maintenance of apical-basal polarity and apical domain development in epithelial cells. ${ }^{4-6}$ Phosphorylation has a key role in controlling polarity, and this is reflected by the fact that Par1 and Par4, homologous in mammals to microtubule affinity regulating kinases (MARKs) and LKB1, respectively, are serine/threonine kinases and Par5 is a member of the 143-3 family of proteins that generally binds Ser/Thr phosphorylated proteins. Additionally, the functions of the Par complex are regulated by phosphorylation. Par3 is phosphorylated on

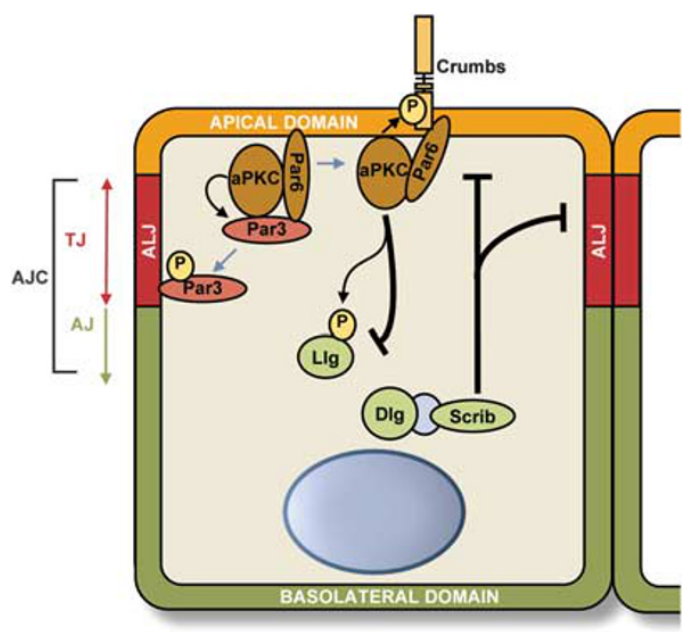

Figure 1 Diagram representing the core polarity components involved in the maintenance of apical-basal polarity in epithelial cells and the establishment of membrane domain identity. AJ, adherens junctions; ALJ, apical/lateral junction; TJ, tight junctions
Ser827 of its aPKC-binding region by aPKC itself, resulting in decreased affinity for aPKC. ${ }^{7}$ Rho kinase also prevents the interaction between Par3 and aPKC by phosphorylating Thr883 of Par3, thereby suppressing the activity of the Par complex. $^{8}$

The activity of the Par complex is further regulated by the dynamic nature of Par3's association with the stable Par6aPKC complex. A compelling study in Drosophila demonstrated that Par3 is in fact excluded from the apical domain by the Par6aPKC complex. ${ }^{9}$ Instead, the Drosophila Par3 homologue Baz localised independently of aPKC and Par6 in the follicular epithelium, and below them at the level of the apical/lateral junction. This correlates with the observation that in many epithelial tissues, including in mammals, Par3 and the Par6aPKC complex do not colocalise. ${ }^{10-13}$ However, in mammals, the apical/lateral domain is formed by tight junctions, which are more apical and distinct from the adherens junctions, thus Par3 is essentially localised at the level of tight junctions where it colocalises with zonula occludens- 1 (ZO-1)..$^{14,15}$ This model for Par3 exclusion from the apical domain involves both the Par6aPKC complex and the Crumbs complex, in order to prevent the interaction between Par3 and the Par6-aPKC complex. On one hand, aPKC phosphorylates Par3 on Ser827 in mammalian Par3 to decrease their affinity for each other while, on the other hand, Crumbs and Stardust compete with Par3 to interact with the same domain of Par6 (Figure 1). This exclusion mechanism is crucial to restrict the extent of the apical/lateral junction and define the border between the apical and lateral domains in Drosophila epithelial cells. Further investigation is required, but the existing evidence suggests that the observations outlined above may be generalised to epithelial tissues in mammals. ${ }^{9}$

The three members of the Scribble complex have been shown to interact genetically, ${ }^{16}$ with $\mathrm{Dlg}$ and Scribble physically interacting through a protein called GUK-holder in Drosophila neuronal synapses. ${ }^{17}$ However, there is little evidence for their physical interaction in mammalian epithelial cells and, as a result, the term 'module' is sometimes used when referring to the Scribble complex. More recently, Scribble and Lgl2 have been reported to interact directly in polarised mammalian epithelial cells, although this interaction has not yet been reported in other experimental systems. ${ }^{18}$ Lgl, by competing with Par3 for Par6-aPKC, restricts the Par complex to the apical domain. ${ }^{5,19}$ Furthermore, phosphorylation and inactivation of $\mathrm{Lgl}$ at the apical domain by aPKC restricts the Par and Scribble complex apically and basolaterally in epithelial cells, respectively. ${ }^{20}$ Interestingly, in Drosophila, aPKC is also able to phosphorylate Crumbs to promote the apical localisation of the Crumbs complex. ${ }^{21}$ Key phosphorylation events and protein/protein interactions therefore result in the exclusion of Scribble from the apical domain, and of the Crumbs and Par complexes from the basolateral domain. These membrane domains consequently acquire unique identities that set the basis for the establishment of apical-basal polarity in epithelial cells.

\section{Cell Polarity and Asymmetric Cell Division}

Asymmetric cell division is pivotal for the maintenance of epithelial tissue homeostasis. When a stem cell divides asymmetrically, it generates two daughter cells: one with an 
identical cell fate and the other with a different one. Asymmetric cell division relies on the asymmetric distribution of cell fate determinants (Numb, Pros, Brat, Pon and Mira) and, as a result, core polarity proteins and the correct orientation of mitotic spindles. 2,22 Emerging evidence, in particular from studies of neuroblasts in Drosophila, indicates that asymmetric division functions as a mechanism of tumour suppression. ${ }^{23}$ When polarity genes or cell fate determinants are deleted or mutated, neuroblasts divide symmetrically, leading to tissue overgrowth and transplantable tumours that are similar to mammalian cancers (Figure 2). For example, $\mathrm{Dlg} / \mathrm{Scrib} / \mathrm{Lgl} \mathrm{mutant}$ neuroblasts have defects in basal protein targeting, a reduced apical cortical domain and reduced apical spindle size, which is thought to lead to symmetric divisions and, as a result, to the accumulation of cells and the development of tumours. ${ }^{24}$ In Drosophila, a number of proteins such as Pins, Mud and the mitotic kinases Aurora-A and Polo have been shown to have a major role in regulating mitotic spindle orientation and asymmetric cell division. ${ }^{25}$ Intriguingly, adenomatous polyposis coli 2 (APC2) was discovered to be part of the centrosome complex of Drosophila germline cells, where it functions in establishing the correct orientation of the mitotic spindles. Moreover, deletion of both Drosophila APC genes results in asymmetric stem cell division defects as a result of mitotic spindle misorientation. ${ }^{26}$ Interestingly, Johnston et al. ${ }^{27}$ have developed an 'induced cortical polarity' assay in Drosophila S2 cells that should facilitate the identification of further proteins, domains and amino acids that regulate spindle orientation.

In mammals, probably as a consequence of their redundancy, the function of core polarity proteins in the regulation of asymmetric cell division has been harder to elucidate. However, Lgl1 knockout mice present some defects in the asymmetric division of neural progenitors, that may result in overproliferation and a lack of differentiation. ${ }^{28}$ Recently, in a compelling study using in vivo electroporation of mouse embryos and cortical slice cultures, Par3 has been shown to regulate the asymmetric division of neural progenitor cells via the control of the Notch signalling pathway in the developing neocortex. $^{29}$ This study has also shown that Par3 is distributed and inherited asymmetrically as cells divide, suggesting that its subcellular distribution regulates the mode of progenitor cell division and daughter cell fate specification.

\section{Polarity and EMT}

The transition from an epithelial to mesenchymal phenotype that occurs during EMT has been associated with metastatic progression; apical-basal polarity is lost during this process and cell-cell junctions are weakened and disrupted. Several lines of evidence suggest that core polarity proteins are important for the formation and maintenance of the AJC, suggesting that their loss could induce or at least contribute to EMT (Figure 3). For example, it has been shown that Par3 depletion in mammalian epithelial cells disrupts the formation of tight junctions. ${ }^{30}$ Moreover, it has been suggested that the Par6-aPKC complex, together with Cdc42, can control adherens junction remodelling through the regulation of Arp2/3-dependent endocytosis. ${ }^{31}$ Another example comes from knock-down experiments showing that PATJ is important for the proper polarisation of mammalian epithelial cells and the formation of tight junctions. ${ }^{32}$ SiRNA-mediated knockdown of Scrib in SK-CO15 cells inhibits tight junction reassembly. ${ }^{33}$

$\operatorname{TGF} \beta$, in cooperation with the Ras pathway, is a key inducer of EMT and promotes invasion and metastasis. Several studies suggest that disrupting cell polarity may work in

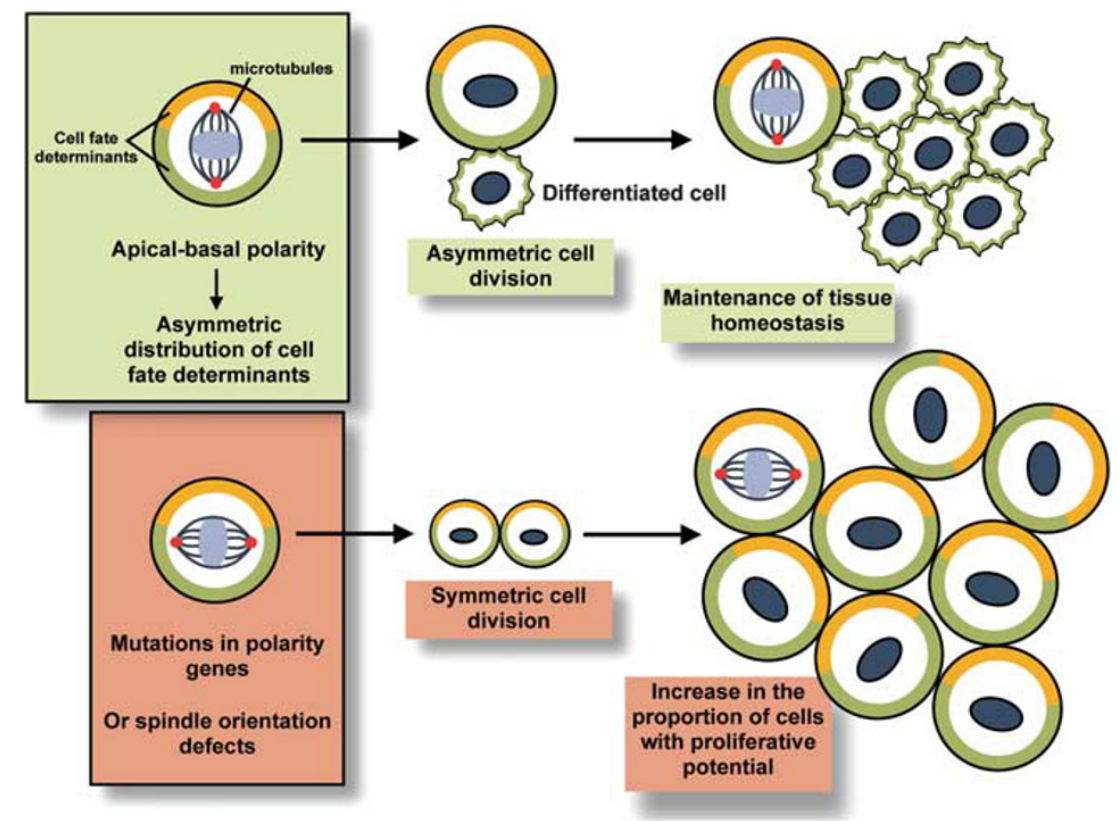

Figure 2 Loss of cell polarity in epithelial stem cells can lead to asymmetric division defects, thereby favouring tumour initiation. Apical-basal polarity is fundamental to the asymmetric segregation of cell fate determinants. Thick yellow and green lines represent cell fate determinants. Red dots represent centrosomes. In the absence of apicalbasal polarity, this segregation is defective, potentially leading to an excess in symmetric divisions and an accumulation of cells with proliferative potential 


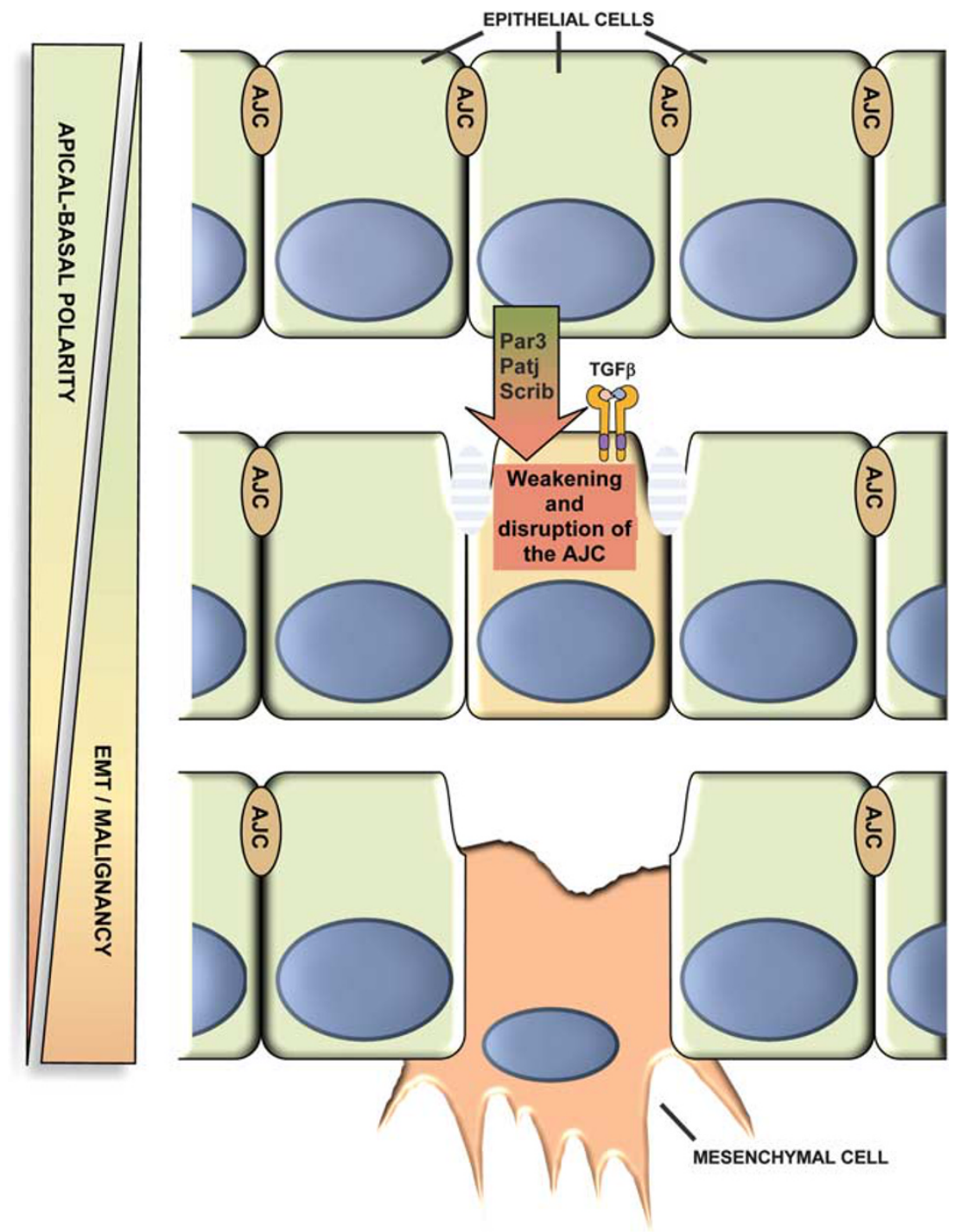

Figure 3 Epithelial cell polarity represents a barrier to the later stages of tumour development. Apical-basal polarity is involved in the formation and maintenance of the AJC. Decreased expression of core polarity proteins is linked to weakening or disruption of the AJC, thereby leading to EMT and potential malignancy. Recent evidence suggests that TGF $\beta$, under certain conditions, can induce EMT in epithelial cells in conjunction with loss of cell polarity

concert with $\operatorname{TGF} \beta$ signalling or facilitate $\operatorname{TGF} \beta$-mediated EMT. For instance, it has recently been shown that disruption of the Crumbs complex predisposes to EMT in Eph4 cells, a cell line normally insensitive to TGF $\beta$-mediated EMT. ${ }^{34}$ Another study shows that TGF $\beta$ signalling is able to downregulate the expression of Par-3 through the induction of miR$491-5 p$ in rat proximal tubular epithelial cells, suggesting that TGF $\beta$ may induce the disruption of cell polarity. ${ }^{35}$ Probably, one of the most direct links between the disruption of cell polarity and EMT comes from the finding that TGF $\beta$ receptors directly interact with Par6 at the level of adherens junctions. ${ }^{36}$ TGF $\beta$ is able to induce phosphorylation of Par6 at Ser345, enabling the recruitment of the E3 ubiquitin ligase Smurf1, which is required for localised TGF $\beta$-induced degradation of the actin regulator RhoA. Importantly, this phosphorylation event is required for TGF $\beta$-dependent EMT in mammary gland epithelial cells, as mutation of this residue blocks EMT and tight junction disruption. Furthermore, in a mammary fat pad assay, over-expression of Par6/S345A in EMT-6 cells reduced their ability to induce metastasis to the lung, further demonstrating the importance of TGF $\beta /$ Par6 signalling in EMT and the development of metastasis. ${ }^{37}$ Taken together, these studies show that polarity proteins are important EMT regulators in epithelial cells.

\section{Is Epithelial Cell Polarity a Gate Keeper Against Cancer?}

Disruption of polarity by activated oncogenes. Underlying their potential role as tumour suppressors, core polarity proteins are often targeted and disrupted by oncogenic signalling. Polarity defects could collaborate with oncogenic pathways to induce tumour formation. For example, Scrib-deficient mutants cooperate with oncogenes to mediate transformation in Drosophila. Normally, Scrib-deficient mutant clones in the eye imaginal discs are eliminated by JNK-dependent apoptosis. 
However, in the presence of activated oncogenic pathways such as Ras or Notch, apoptosis is inhibited and neoplastic tumours occur. ${ }^{38}$ In addition, in support of these data, loss of hScrib has been shown to cooperate with $\mathrm{H}$-Ras to promote cell invasion through deregulation of MAPK signalling in an organotypic culture system. ${ }^{39}$

A number of viral oncogenes have been found to directly interact with polarity proteins, suggesting that disruption of polarity is important. ${ }^{40}$ For example, the human T-cell leukemia virus type 1 tax protein binds to hScrib and alters its subcellular localisation in infected T-cells. ${ }^{41,42}$ In addition to Scrib, Tax and the oncoprotein 9ORF1 have been shown to interact with Dlg. ${ }^{43-45}$ The interaction of Tax and hDlg was shown to affect the function of hDlg in controlling cell growth, and Tax disrupted the interaction between hDIg and APC. ${ }^{46}$ However, it remains to be shown whether disruption of the hDIg/APC complex is the critical event that leads to deregulated cell growth. E6 oncogenes in human papilloma virus (HPV) have also been found to interact with PDZcontaining polarity proteins. For example, E6 proteins from HPV-16 and HPV-18 have the ability to interact with, and induce the proteasomal degradation of, DIg and hScrib. ${ }^{47}$ Intriguingly, E6 has also been shown to target phosphorylated forms of Dig, and Patj of the Crumbs polarity complex, for degradation. ${ }^{48,49}$ These findings suggest that key PDZcontaining polarity proteins are common cellular targets of viral oncoproteins. Moreover, it seems that viral proteins have evolved additional ways to target cell polarity, strongly suggesting that its disruption is necessary for malignant transformation. ${ }^{40}$

In addition to viral oncogenes, core cell polarity mechanisms are targeted by abnormally activated growth factor signalling pathways. Both the ErbB2 and TGF $\beta$ signalling pathways have been shown to be directly involved in the regulation of polarity independently of their transcriptional response. $^{36,50}$ In conclusion, many activated oncogenic pathways target and disrupt epithelial polarity in order to achieve malignant transformation, supporting the importance of cell polarity in suppressing tumour formation.

\footnotetext{
The core polarity proteins as tumour suppressors in mammals. Scrib, dlg and Igl were identified as tumour suppressors in Drosophila, in screens for mutations causing cancerous overgrowth of the larval imaginal discs and brain. ${ }^{51}$. Interestingly, the phenotypes of these mutants can be rescued by the mammalian homologues of these genes, showing that they are functionally conserved and suggesting that they may have a tumour suppressive function in human cells. ${ }^{52-55}$ A growing amount of data showing mislocalisation, decreased expression or complete loss of the products of these genes in primary tumours from human patients further indicate their involvement in mammalian tumourigenesis. ${ }^{56}$ Over the past few years, there has been an increasing body of evidence that the deregulation of these core polarity complexes, both in terms of expression level and subcellular localisation, may have a causal link to disease and cancer in particular (for an exhaustive list of changes in expression and localisation of polarity proteins in carcinoma cell lines and primary tissues see Huang and Muthuswamy ${ }^{56}$ ).
}

Perhaps the best-studied polarity complex in human tumours is the Scrib/Lgl/Dlg complex. In particular, the deregulation of Scrib has been reported to promote the transformation of mammary epithelial cells in vitro and in vivo, by disrupting morphogenesis and cell polarity and by inhibiting myc-induced apoptosis, thus providing novel insight into how core polarity proteins regulate cell transformation. ${ }^{57}$ In this study, loss of Scrib was shown to cooperate with Myc to induce mammary tumours, correlating with a Drosophila study that showed that Igl requires Myc to promote clonal malignancy. ${ }^{58}$ In human keratinocytes, Scrib loss has been shown to result in ERK activation which might contribute to cancer progression. ${ }^{59}$ Scrib was found to be mislocalised or downregulated in several cancer types including cervical, ${ }^{60}$ colon adenocarcinoma ${ }^{61}$ and endometrial. ${ }^{62} \mathrm{Scrib}$, as well as Dlg1 and Lgl1, was also mislocalised and downregulated in a transgenic mouse model of cancer. ${ }^{63}$ In a screen of 60 tumour samples, Hugl-1 (the human homologue of Lgl) transcripts were often reduced or lost in tumour tissues of the breast (76\%), prostate (53\%), lung (63\%), ovarian $(50 \%)$ and colon carcinoma $(75 \%) .{ }^{53}$ However, the numbers in this study are relatively small and it will be important to verify these data on a larger scale. Hugl-1 was lost in $75 \%$ of tumour samples in a cohort of 94 patients undergoing surgery for colorectal cancer, and was associated with advanced stage and lymph node metastases. ${ }^{64}$ Loss or downregulation of Hugl-1 expression was also observed in malignant melanoma and associated with an advanced stage of the disease. ${ }^{54} \mathrm{~A}$ study of 80 hepatocellular carcinomas showed that Hugl-1 mRNA is frequently mutated by aberrant splicing, and that two of the variants were able to promote hepatocellular carcinoma in nude mice. ${ }^{65}$ Dig expression levels and localisation have also been shown to be affected in high-grade premalignant cervical neoplasia, invasive squamous cell carcinoma ${ }^{66}$ and colon adenocarcinoma. $^{61}$

The deregulation of core polarity proteins in cancer is not limited to Scrib, DIg and Lgl, however, as almost every protein involved in the core apical-basal polarity machinery of epithelial cells has been shown to be affected in some way. ${ }^{56}$ For example, the par3 gene is deleted in $15 \%$ of primary oesophageal squamous cell carcinomas and downregulated in a number of tumour tissues. ${ }^{67} \mathrm{~A}$ recent study identified homozygous intragenic microdeletions, involving genes encoding components of the core polarity complexes in a genome-wide screen of 684 cell lines. ${ }^{68}$ Interestingly, among these genes, Par3 was found to be the most commonly targeted and was disrupted in both cell lines and some primary tumours. This study will undoubtedly be complemented by data from ongoing cancer genome sequencing efforts such as the Cancer Genome Atlas. The Par6-aPKC complex has also been shown to be deregulated in cancer. For example, deregulation of Par6 was observed in ER-positive breast tumours. ${ }^{69}$ Taken together, these data strongly underline the causal link between the deregulation of core polarity proteins and human cancer.

Tumour suppressors that regulate epithelial cell polarity. Over the past decade, a number of tumour suppressor pathways have been directly linked to epithelial cell polarity, suggesting that the integrity of apical-basal 
polarity is crucial for the prevention of tumour development. Importantly, one such tumour suppressor is Par4/LKB1. Although LKB1 deficiency does not cause a gross defect in cell polarity in the intestine, for example, ${ }^{70}$ it may regulate cell polarity through its ability to phosphorylate members of the AMPK-related kinase (ARK) family in other tissues. ${ }^{71,72}$ Hence, Par4/LKB1 is able to activate Par1/MARK in the Drosophila oocyte and, in epithelial cells, may have its role through Par1/ MARK activation. ${ }^{73}$ Alternatively, Par4/LKB1 may regulate polarity through the activation of AMPK (AMP-dependent activated protein kinase), which in turn activates Myosin II. $^{73}$ Among all of the Par genes identified in model organisms, Par4/ LKB1 is the most well-established tumour suppressor. Germline mutations in the $L K B 1$ gene cause Peutz-Jeghers syndrome and predispose patients to develop colon cancer. ${ }^{74,75}$ All of these factors suggest that regulators of cell polarity may have an important role in suppressing tumours. In agreement with this, a growing list of tumour suppressors has been identified that regulate cell polarity. For example, the tumour suppressor von Hippel-Lindau exerts its regulation on polarity at several levels: it is able to directly interact with aPKC and mediate its ubiquitination and subsequent degradation, and its interaction with the Par complex is involved in the regulation of polarised microtubule growth and formation of primary cilia. ${ }^{76,77}$ Another tumour suppressor that regulates cell polarity is phosphatase and tensin homolog (PTEN), which is likely to be involved in different aspects of epithelial cell polarity. However, as apical accumulation of phosphatidylinositol 4,5 biphosphate is dependent on apical targeting of PTEN, and as membrane targeting of Par3 is mediated by direct binding to phosphoinositide lipids, PTEN may be instrumental in the apical localisation of Par3. ${ }^{78-80}$ Interestingly, APC has been shown to interact with several core polarity proteins. For example, hDlg can interact with the APC tumour suppressor, and their interaction negatively regulates cell cycle progression. ${ }^{81}$ APC was also found to interact with hScrib and it has been suggested that Scrib controls its localisation at the level of the adherens junctions in epithelial cells. ${ }^{82}$

Recently, apical-basal polarity in epithelial cells has been linked to another tumour suppressor, with the discovery that ASPP2 is a new binding partner of Par3. ${ }^{83,84}$ ASPP2 is critical for the localisation of Par3 at the level of tight junctions in epithelial cells and, therefore, has a crucial role in the establishment and maintenance of apical-basal polarity in epithelial cells in culture. In vivo, ASPP2 deficiency results in defects arising during the development of the central nervous system, characterised by a loss of apical-basal polarity and an expansion of neural progenitor cells. ${ }^{84}$ ASPP2 colocalises with Par3 at the level of tight junctions in a variety of epithelial cells and tissues, suggesting that its role in controlling apicalbasal polarity is common to other epithelia. ${ }^{83,84}$ ASPP2 is a transcriptional target of $\mathrm{E} 2 \mathrm{~F} 1^{85}$ and was first identified as a p53 regulator that specifically promotes its apoptotic function. ${ }^{86}$ Tumour studies in mice have identified ASPP2 as a haploinsufficient tumour suppressor gene, ${ }^{87,88}$ and ASPP2 levels have been shown to be deregulated in human tumours and tumour cell lines, suggesting that its tumour suppressive role is conserved in humans. ${ }^{89-91}$ Studies in Drosophila have shown that dASPP, the unique ASPP protein in Drosophila, localises at adherens junctions. These studies suggest that
dASPP regulates retinal morphogenesis by acting in concert with dRASSF8 to promote dCSK activity, thus, the function of ASPP2 in the establishment and maintenance of apical-basal polarity in epithelial cells appears to have been conserved from Drosophila to humans. ${ }^{92,93}$ However, it remains unclear whether the function of ASPP2 in regulating apical-basal polarity is linked to its ability to regulate p53 and its family members, p63 and p73. One possibility is that ASPP2, upon external stimuli, may be able to shuttle from tight junctions to the nucleus to have its transcriptional role, in a similar way to other junctional proteins such as $\beta$-catenin or ZO-1. Therefore, it will be of great interest to investigate the extent to which ASPP2's regulation of the localisation of Par3 and the apicalbasal polarity of epithelial cells contributes to its tumour suppressive function, and how this interplays with its role in regulating $\mathrm{p53}$, the most mutated tumour suppressor in human cancers, and p63, a key regulator of epithelial stratification.

There are a number of studies that also suggest that mitotic spindle orientation is crucial for asymmetric cell division in mammals. For instance, p63 has been shown to be important for mitotic spindle orientation during asymmetric cell division of epidermal stem cells. ${ }^{94}$ Finally, emerging evidence suggests that there is also a direct relationship between loss of cell polarity of stem cells and asymmetric division and tumour initiation in mammals. For example, the tumour suppressor p53 has recently been linked with the regulation of asymmetric divisions of mammary stem cells. ${ }^{95}$ In the future, due to their redundancy in mammals, the real challenge will be to investigate whether core polarity proteins regulate asymmetric stem cell division in mammalian epithelia, and whether their deregulation consequently drives tumour initiation as a result of asymmetric stem cell division defects.

Taken together, these studies emphasise the role and importance of known tumour suppressors in the control of epithelial cell polarity. Many of those tumour suppressors regulate the functions of core polarity proteins through direct interactions suggesting that, in addition to their better-known roles in the control of cellular proliferation, these roles are crucial for the prevention of tumour development.

\section{Conclusion}

In this review, we have highlighted how epithelial cell polarity may contribute to tumour suppression (Figure 4), through its role in controlling asymmetric cell division and the integrity of the AJC. Loss of cell polarity is a hallmark of cancer, however, studies in transgenic mouse models have so far been unable to clearly answer the question of whether core polarity proteins are tumour suppressors or not. Future studies using multiple and/or conditional knockout mouse models will be essential to finally demonstrate their direct role in tumour suppression. Due to the vital importance of core polarity proteins in maintaining tissue homeostasis, redundancy mechanisms have evolved in mammals that may make this issue too complex to address. Nonetheless, it is emerging that an increasing number of well-known tumour suppressors have a pivotal role in regulating cell polarity. Hence, regulators of polarity may themselves represent a new class of tumour suppressors. 


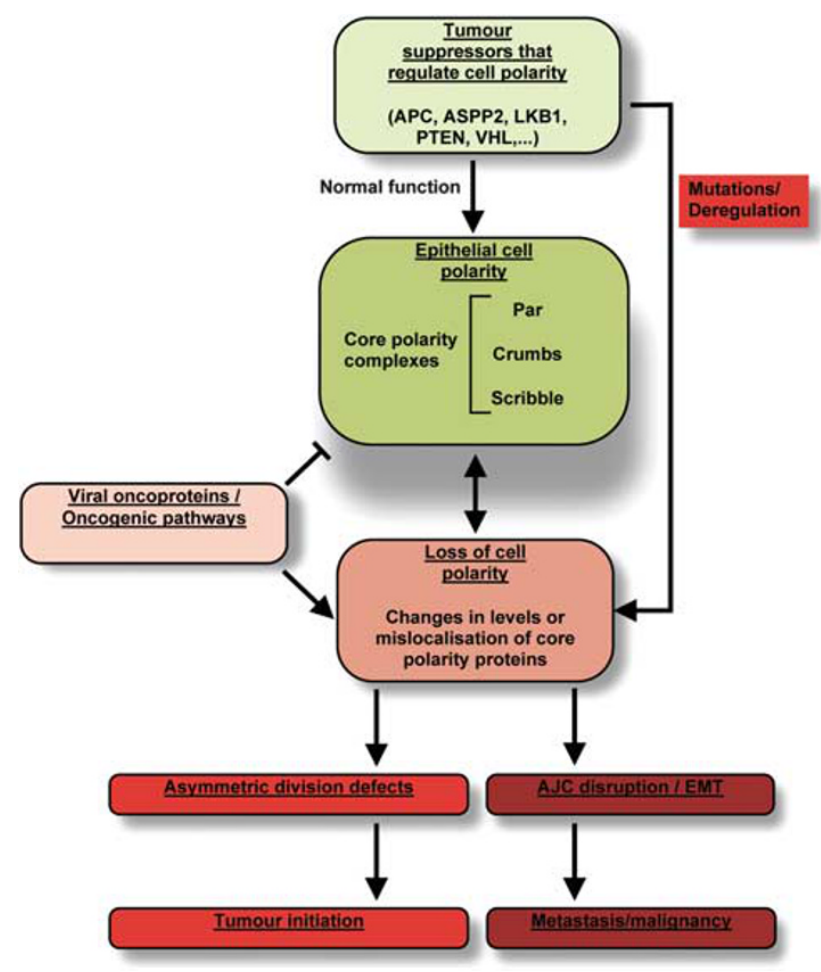

Figure 4 A summary of epithelial cell polarity's role in suppressing tumour initiation and malignancy

\section{Conflict of Interest}

The authors declare no conflict of interest.

1. Humbert PO, Grzeschik NA, Brumby AM, Galea R, Elsum I, Richardson HE. Control of tumourigenesis by the Scribble/Dlg/Lgl polarity module. Oncogene 2008; 27: 6888-6907.

2. Lee M, Vasioukhin V. Cell polarity and cancer-cell and tissue polarity as a non-canonical tumor suppressor. J Cell Sci 2008; 121 (Part 8): 1141-1150.

3. Medina E, Lemmers C, Lane-Guermonprez L, Le Bivic A. Role of the Crumbs complex in the regulation of junction formation in Drosophila and mammalian epithelial cells. Biol Cell/ Under Aus Eu Cell Biol Organization 2002; 94: 305-313.

4. Hirose T, Izumi Y, Nagashima Y, Tamai-Nagai $Y$, Kurihara H, Sakai T et al. Involvement of ASIP/PAR-3 in the promotion of epithelial tight junction formation. J Cell Sci 2002; 115 (Part 12): 2485-2495.

5. Hutterer A, Betschinger J, Petronczki M, Knoblich JA. Sequential roles of Cdc42, Par-6, $\mathrm{aPKC}$, and $\mathrm{Lgl}$ in the establishment of epithelial polarity during drosophila embryogenesis. Dev Cell 2004; 6: 845-854.

6. Horikoshi Y, Suzuki A, Yamanaka T, Sasaki K, Mizuno K, Sawada $\mathrm{H}$ et al. Interaction between PAR-3 and the aPKC-PAR-6 complex is indispensable for apical domain development of epithelial cells. J Cell Sci 2009; 122 (Part 10): 1595-1606.

7. Nagai-Tamai Y, Mizuno K, Hirose T, Suzuki A, Ohno S. Regulated protein-protein interaction between aPKC and PAR-3 plays an essential role in the polarization of epithelia cells. Genes Cells 2002; 7: 1161-1171.

8. Nakayama M, Goto TM, Sugimoto M, Nishimura T, Shinagawa T, Ohno S et al. Rho-kinase phosphorylates PAR-3 and disrupts PAR complex formation. Dev Cell 2008; 14: 205-215.

9. Morais-de-Sa E, Mirouse V, St Johnston D. aPKC phosphorylation of Bazooka defines the apical/lateral border in drosophila epithelial cells. Cell 2010; 141: 509-523.

10. Harris TJ, Peifer M. The positioning and segregation of apical cues during epithelial polarity establishment in Drosophila. J Cell Biol 2005; 170: 813-823.

11. Martin-Belmonte F, Gassama A, Datta A, Yu W, Rescher U, Gerke V et al. PTEN-mediated apical segregation of phosphoinositides controls epithelial morphogenesis through $\mathrm{Cdc} 42$. Cell 2007; 128: 383-397.

12. Nam SC, Choi KW. Interaction of Par-6 and crumbs complexes is essential for photoreceptor morphogenesis in drosophila. Development 2003; 130: 4363-4372.

13. Vogelmann R, Nelson WJ. Fractionation of the epithelial apical junctional complex: reassessment of protein distributions in different substructures. Mol Biol Cell 2005; 16 701-716.
14. Izumi $Y$, Hirose $T$, Tamai $Y$, Hirai $S$, Nagashima $Y$, Fujimoto $T$ et al. An atypical PKC directly associates and colocalizes at the epithelial tight junction with ASIP, a mammalian homologue of Caenorhabditis elegans polarity protein PAR-3. J Cell Biol 1998; 143: 95-106.

15. Joberty G, Petersen C, Gao L, Macara IG. The cell-polarity protein Par6 links Par3 and atypical protein kinase $\mathrm{C}$ to Cdc42. Nat Cell Biol 2000; 2: 531-539.

16. Bilder D, Li M, Perrimon N. Cooperative regulation of cell polarity and growth by Drosophila tumor suppressors. Science 2000; 289: 113-116.

17. Mathew D, Gramates LS, Packard M, Thomas U, Bilder D, Perrimon N et al. Recruitment of scribble to the synaptic scaffolding complex requires GUK-holder, a novel DLG binding protein. Curr Biol 2002; 12: 531-539.

18. Kallay LM, McNickle A, Brennwald PJ, Hubbard AL, Braiterman LT. Scribble associates with two polarity proteins, Lgl2 and Vangl2, via distinct molecular domains. J Cell Biochem 2006; 99: 647-664.

19. Yamanaka T, Horikoshi Y, Izumi N, Suzuki A, Mizuno K, Ohno S. Lgl mediates apical domain disassembly by suppressing the PAR-3-aPKC-PAR- 6 complex to orient apical membrane polarity. J Cell Sci 2006; 119 (Part 10): 2107-2118.

20. Plant PJ, Fawcett JP, Lin DC, Holdorf AD, Binns K, Kulkarni S et al. A polarity complex of mPar-6 and atypical PKC binds, phosphorylates and regulates mammalian Lgl. Nat Cell Biol 2003; 5: 301-308.

21. Sotillos S, Diaz-Meco MT, Caminero E, Moscat J, Campuzano S. DaPKC-dependent phosphorylation of crumbs is required for epithelial cell polarity in drosophila. J Cell Bio 2004; 166: 549-557.

22. Knoblich JA. Asymmetric cell division: recent developments and their implications for tumour biology. Nat Rev Mol Cell Biol 2010; 11: 849-860.

23. Januschke J, Gonzalez C. Drosophila asymmetric division, polarity and cancer. Oncogene 2008; 27: 6994-7002.

24. Albertson R, Doe CQ. Dlg, Scrib and Lgl regulate neuroblast cell size and mitotic spindle asymmetry. Nat Cell Biol 2003; 5: 166-170.

25. Chia W, Somers WG, Wang H. Drosophila neuroblast asymmetric divisions: cell cycle regulators, asymmetric protein localization, and tumorigenesis. J Cell Biol 2008; 180 : 267-272.

26. Yamashita YM, Jones DL, Fuller MT. Orientation of asymmetric stem cell division by the APC tumor suppressor and centrosome. Science 2003; 301: 1547-1550.

27. Johnston CA, Hirono K, Prehoda KE, Doe CQ. Identification of an Aurora-A/PinsLINKER/ Dlg spindle orientation pathway using induced cell polarity in S2 cells. Cell 2009; 138 $1150-1163$.

28. Klezovitch O, Fernandez TE, Tapscott SJ, Vasioukhin V. Loss of cell polarity causes severe brain dysplasia in Lgl1 knockout mice. Genes Dev 2004; 18: 559-571.

29. Bultje RS, Castaneda-Castellanos DR, Jan LY, Jan YN, Kriegstein AR, Shi SH Mammalian Par3 regulates progenitor cell asymmetric division via notch signaling in the developing neocortex. Neuron 2009; 63: 189-202.

30. Chen X, Macara IG. Par-3 controls tight junction assembly through the Rac exchange factor Tiam1. Nat Cell Biol 2005; 7: 262-269.

31. Georgiou M, Marinari E, Burden J, Baum B. Cdc42, Par6, and aPKC regulate Arp2/3-mediated endocytosis to control local adherens junction stability. Curr Biol 2008; 18: 1631-1638.

32. Shin K, Straight S, Margolis B. PATJ regulates tight junction formation and polarity in mammalian epithelial cells. J Cell Biol 2005; 168: 705-711.

33. Ivanov Al, Young C, Den Beste K, Capaldo CT, Humbert PO, Brennwald P et al. Tumor suppressor scribble regulates assembly of tight junctions in the intestinal epithelium. $A m$ Pathol 2010; 176: 134-145.

34. Varelas X, Samavarchi-Tehrani P, Narimatsu M, Weiss A, Cockburn K, Larsen BG et al. The Crumbs complex couples cell density sensing to Hippo-dependent control of the TGFbeta-SMAD pathway. Dev Cell 2010; 19: 831-844.

35. Zhou Q, Fan J, Ding X, Peng W, Yu X, Chen Y et al. TGF-\{beta\}-induced MiR-491-5p expression promotes Par-3 degradation in rat proximal tubular epithelial cells. J Biol Chem 2010; 285: 40019-40027.

36. Ozdamar B, Bose R, Barrios-Rodiles M, Wang HR, Zhang Y, Wrana JL. Regulation of the polarity protein Par6 by TGFbeta receptors controls epithelial cell plasticity. Science 2005 307: 1603-1609.

37. Viloria-Petit AM, David L, Jia JY, Erdemir T, Bane AL, Pinnaduwage D et al. A role for the TGFbeta-Par6 polarity pathway in breast cancer progression. Proc Natl Acad Sci USA 2009; 106: 14028-14033.

38. Brumby AM, Richardson HE. Scribble mutants cooperate with oncogenic Ras or Notch to cause neoplastic overgrowth in drosophila. EMBO J 2003; 22: 5769-5779.

39. Dow LE, Elsum IA, King CL, Kinross KM, Richardson HE, Humbert PO. Loss of human scribble cooperates with $\mathrm{H}$-Ras to promote cell invasion through deregulation of MAPK signalling. Oncogene 2008; 27: 5988-6001.

40. Javier RT. Cell polarity proteins: common targets for tumorigenic human viruses. Oncogene 2008; 27: 7031-7046.

41. Okajima M, Takahashi M, Higuchi M, Ohsawa T, Yoshida S, Yoshida Y et al. Human T-cel leukemia virus type 1 Tax induces an aberrant clustering of the tumor suppressor scribble through the PDZ domain-binding motif dependent and independent interaction. Virus Genes 2008; 37: 231-240.

42. Arpin-Andre C, Mesnard JM. The PDZ domain-binding motif of the human T cell leukemia virus type 1 tax protein induces mislocalization of the tumor suppressor hScrib in T cells. J Biol Chem 2007; 282: 33132-33141. 
43. Lee SS, Weiss RS, Javier RT. Binding of human virus oncoproteins to hDlg/SAP97, a mammalian homolog of the drosophila discs large tumor suppressor protein. Proc Natl Acad Sci USA 1997; 94: 6670-6675.

44. Kanamori M, Sandy P, Marzinotto S, Benetti R, Kai C, Hayashizaki Y et al. The PDZ protein tax-interacting protein-1 inhibits beta-catenin transcriptional activity and growth of colorectal cancer cells. J Biol Chem 2003; 278: 38758-38764.

45. Hirata A, Higuchi M, Niinuma A, Ohashi M, Fukushi M, Oie M et al. PDZ domain-binding motif of human T-cell leukemia virus type 1 Tax oncoprotein augments the transforming activity in a rat fibroblast cell line. Virology 2004; 318: 327-336.

46. Suzuki T, Ohsugi Y, Uchida-Toita M, Akiyama T, Yoshida M. Tax oncoprotein of HTLV-1 binds to the human homologue of drosophila discs large tumor suppressor protein, hDLG and perturbs its function in cell growth control. Oncogene 1999; 18: 5967-5972.

47. Thomas M, Massimi P, Navarro C, Borg JP, Banks L. The hScrib/DIg apico-basal control complex is differentially targeted by HPV-16 and HPV-18 E6 proteins. Oncogene 2005; 24 : 6222-6230.

48. Storrs $\mathrm{CH}$, Silverstein SJ. PATJ, a tight junction-associated PDZ protein, is a nove degradation target of high-risk human papillomavirus E6 and the alternatively spliced isoform $18 \mathrm{E6}$. J Virol 2007; 81: 4080-4090.

49. Narayan N, Subbaiah VK, Banks L. The high-risk HPV E6 oncoprotein preferentially targets phosphorylated nuclear forms of hDlg. Virology 2009; 387: 1-4

50. Aranda V, Haire T, Nolan ME, Calarco JP, Rosenberg AZ, Fawcett JP et al. Par6-aPKC uncouples ErbB2 induced disruption of polarized epithelial organization from proliferation control. Nat Cell Biol 2006; 8: 1235-1245.

51. Gateff E. Malignant neoplasms of genetic origin in Drosophila melanogaster. Science 1978; 200: 1448-1459.

52. Dow LE, Brumby AM, Muratore R, Coombe ML, Sedelies KA, Trapani JA et al. hScrib is a functional homologue of the Drosophila tumour suppressor Scribble. Oncogene 2003; 22 9225-9230.

53. Grifoni D, Garoia F, Schimanski CC, Schmitz G, Laurenti E, Galle PR et al. The human protein $\mathrm{Hugl}-1$ substitutes for drosophila lethal giant larvae tumour suppressor function in vivo. Oncogene 2004; 23: 8688-8694.

54. Kuphal S, Wallner S, Schimanski CC, Bataille F, Hofer P, Strand S et al. Expression of Hugl-1 is strongly reduced in malignant melanoma. Oncogene 2006; 25: 103-110.

55. Thomas U, Phannavong B, Muller B, Garner CC, Gundelfinger ED. Functional expression of rat synapse-associated proteins SAP97 and SAP102 in Drosophila dlg-1 mutants: effects on tumor suppression and synaptic bouton structure. Mech Dev 1997; 62: 161-174.

56. Huang L, Muthuswamy SK. Polarity protein alterations in carcinoma: a focus on emerging roles for polarity regulators. Curr Opin Genet Dev 2010; 20: 41-50.

57. Zhan L, Rosenberg A, Bergami KC, Yu M, Xuan Z, Jaffe AB et al. Deregulation of scribble promotes mammary tumorigenesis and reveals a role for cell polarity in carcinoma. Cell 2008; 135: 865-878.

58. Froldi F, Ziosi M, Garoia F, Pession A, Grzeschik NA, Bellosta $P$ et al. The lethal giant larvae tumour suppressor mutation requires dMyc oncoprotein to promote clonal malignancy. BMC Biol 2010; 8: 33.

59. Nagasaka K, Pim D, Massimi P, Thomas M, Tomaic V, Subbaiah VK et al. The cell polarity regulator hScrib controls ERK activation through a KIM site-dependent interaction. Oncogene 2010; 29: 5311-5321.

60. Nakagawa S, Yano T, Nakagawa K, Takizawa S, Suzuki Y, Yasugi T et al. Analysis of the expression and localisation of a LAP protein, human scribble, in the normal and neoplastic epithelium of uterine cervix. Br J Cancer 2004; 90: 194-199.

61. Gardiol D, Zacchi A, Petrera F, Stanta G, Banks L. Human discs large and scrib are localized at the same regions in colon mucosa and changes in their expression patterns are correlated with loss of tissue architecture during malignant progression. Int J Cancer 2006 119: $1285-1290$.

62. Ouyang Z, Zhan W, Dan L. hScrib, a human homolog of Drosophila neoplastic tumo suppressor, is involved in the progress of endometrial cancer. Oncol Res 2010; 18: 593-599.

63. Vieira V, de la Houssaye G, Lacassagne E, Dufier JL, Jais JP, Beermann F et al. Differential regulation of Dlg1, Scrib, and Lgl1 expression in a transgenic mouse model of ocular cancer. Mol Vis 2008; 14: 2390-2403.

64. Schimanski CC, Schmitz G, Kashyap A, Bosserhoff AK, Bataille F, Schafer SC et al. Reduced expression of Hugl-1, the human homologue of Drosophila tumour suppressor gene Igl, contributes to progression of colorectal cancer. Oncogene 2005; 24: 3100-3109.

65. Lu X, Feng X, Man X, Yang G, Tang L, Du D et al. Aberrant splicing of Hugl-1 is associated with hepatocellular carcinoma progression. Clin Cancer Res 2009; 15: 3287-3296.

66. Lin HT, Steller MA, Aish L, Hanada T, Chishti AH. Differential expression of human Dlg in cervical intraepithelial neoplasias. Gynecol Oncol 2004; 93: 422-428.

67. Zen K, Yasui K, Gen Y, Dohi O, Wakabayashi N, Mitsufuji S et al. Defective expression of polarity protein PAR-3 gene (PARD3) in esophageal squamous cell carcinoma. Oncogene 2009; 28: 2910-2918.

68. Rothenberg SM, Mohapatra G, Rivera MN, Winokur D, Greninger P, Nitta M et al. A genome-wide screen for microdeletions reveals disruption of polarity complex genes in diverse human cancers. Cancer Res 2010; 70: 2158-2164.

69. Nolan ME, Aranda V, Lee S, Lakshmi B, Basu S, Allred DC et al. The polarity protein Par6 induces cell proliferation and is overexpressed in breast cancer. Cancer Res 2008; 68: 8201-8209.
70. Shorning BY, Zabkiewicz J, McCarthy A, Pearson HB, Winton DJ, Sansom OJ et al. Lkb1 deficiency alters goblet and paneth cell differentiation in the small intestine. PloS One 2009; 4: e4264.

71. Pearson HB, McCarthy A, Collins CM, Ashworth A, Clarke AR. Lkb1 deficiency causes prostate neoplasia in the mouse. Cancer Res 2008; 68: 2223-2232.

72. Hezel AF, Gurumurthy S, Granot Z, Swisa A, Chu GC, Bailey G et al. Pancreatic LKB1 deletion leads to acinar polarity defects and cystic neoplasms. Mol Cell Biol 2008; 28: 2414-2425.

73. Mirouse V, Billaud M. The LKB1/AMPK polarity pathway. FEBS Lett 2010; 585: 981-986.

74. Hemminki A, Markie D, Tomlinson I, Avizienyte E, Roth S, Loukola A et al. A serine/ threonine kinase gene defective in Peutz-Jeghers syndrome. Nature 1998; 391: 184-187.

75. Jenne DE, Reimann $\mathrm{H}$, Nezu J, Friedel W, Loff S, Jeschke $\mathrm{R}$ et al. Peutz-Jeghers syndrome is caused by mutations in a novel serine threonine kinase. Nat Genet 1998; 18: 38-43.

76. Okuda H, Saitoh K, Hirai S, Iwai K, Takaki Y, Baba M et al. The von Hippel-Lindau tumor suppressor protein mediates ubiquitination of activated atypical protein kinase C. J Biol Chem 2001; 276: 43611-43617.

77. Schermer B, Ghenoiu C, Bartram M, Muller RU, Kotsis F, Hohne M et al. The von HippelLindau tumor suppressor protein controls ciliogenesis by orienting microtubule growth. $J$ Cell Biol 2006; 175: 547-554.

78. Feng W, Wu H, Chan LN, Zhang M. Par-3-mediated junctional localization of the lipid phosphatase PTEN is required for cell polarity establishment. J Biol Chem 2008; 283: 23440-23449.

79. Krahn MP, Klopfenstein DR, Fischer N, Wodarz A. Membrane targeting of Bazooka/PAR-3 is mediated by direct binding to phosphoinositide lipids. Curr Biol 2010; 20: 636-642.

80. Wu H, Feng W, Chen J, Chan LN, Huang S, Zhang M. PDZ domains of Par-3 as potential phosphoinositide signaling integrators. Mol Cell 2007; 28: 886-898.

81. Ishidate $\mathrm{T}$, Matsumine A, Toyoshima K, Akiyama T. The APC-hDLG complex negatively regulates cell cycle progression from the G0/G1 to S phase. Oncogene 2000; 19: $365-372$.

82. Takizawa S, Nagasaka K, Nakagawa S, Yano T, Nakagawa K, Yasugi T et al. Human scribble, a novel tumor suppressor identified as a target of high-risk HPV E6 for ubiquitinmediated degradation, interacts with adenomatous polyposis coli. Genes Cells 2006; 11: 453-464.

83. Cong W, Hirose T, Harita Y, Yamashita A, Mizuno K, Hirano $\mathrm{H}$ et al. ASPP2 regulates epithelial cell polarity through the PAR complex. Curr Biol 2010; 20: 1408-1414.

84. Sottocornola R, Royer C, Vives V, Tordella L, Zhong S, Wang Y et al. ASPP2 binds Par-3 and controls the polarity and proliferation of neural progenitors during CNS development. Dev Cell 2010; 19: 126-137.

85. Fogal V, Kartasheva NN, Trigiante G, Llanos S, Yap D, Vousden KH et al. ASPP1 and ASPP2 are new transcriptional targets of E2F. Cell Death Differ 2005; 12: 369-376

86. Samuels-Lev Y, O'Connor DJ, Bergamaschi D, Trigiante G, Hsieh JK, Zhong S et al. ASPP proteins specifically stimulate the apoptotic function of p53. Mol Cell 2001; 8: 781-794.

87. Kampa KM, Acoba JD, Chen D, Gay J, Lee H, Beemer K et al. Apoptosis-stimulating protein of p53 (ASPP2) heterozygous mice are tumor-prone and have attenuated cellular damage-response thresholds. Proc Natl Acad Sci USA 2009; 106: 4390-4395.

88. Vives V, Su J, Zhong S, Ratnayaka I, Slee E, Goldin R et al. ASPP2 is a haploinsufficient tumor suppressor that cooperates with p53 to suppress tumor growth. Genes Dev 2006; 20: $1262-1267$.

89. Liu WK, Jiang XY, Ren JK, Zhang ZX. Expression pattern of the ASPP family members in endometrial endometrioid adenocarcinoma. Onkologie 2010; 33: 500-503.

90. Liu ZJ, Lu X, Zhang Y, Zhong S, Gu SZ, Zhang XB et al. Downregulated mRNA expression of ASPP and the hypermethylation of the $5^{\prime}$-untranslated region in cancer cell lines retaining wild-type p53. FEBS Lett 2005; 579: 1587-1590.

91. Park SW, An CH, Kim SS, Yoo NJ, Lee SH. Mutational analysis of ASPP1 and ASPP2 genes, a p53-related gene, in gastric and cololorectal cancers with microsatellite instability. Gut Liver 2010; 4: 292-293.

92. Langton PF, Colombani J, Aerne BL, Tapon N. Drosophila ASPP regulates C-terminal Src kinase activity. Dev Cell 2007; 13: 773-782.

93. Langton PF, Colombani J, Chan EH, Wepf A, Gstaiger M, Tapon N. The dASPP-dRASSF8 complex regulates cell-cell adhesion during drosophila retinal morphogenesis. Curr Biol 2009; 19: 1969-1978

94. Lechler T, Fuchs E. Asymmetric cell divisions promote stratification and differentiation of mammalian skin. Nature 2005; 437: 275-280.

95. Cicalese A, Bonizzi G, Pasi CE, Faretta M, Ronzoni S, Giulini B et al. The tumor suppressor p53 regulates polarity of self-renewing divisions in mammary stem cells. Cell 2009; 138: 1083-1095

This work is licensed under the Creative Commons Attribution-NonCommercial-Share Alike 3.0 Unported License. To view a copy of this license, visit http://creativecommons. org/licenses/by-nc-sa/3.0/ 